\title{
Practice environment related barriers to exclusive breastfeeding among HIV-positive mothers in Blantyre, Malawi
}

\author{
Ursula K. Kafulafula ${ }^{1 *}$, Mary K. Hutchinson ${ }^{2}$, Susan Gennaro ${ }^{3}$, Sally Guttmacher ${ }^{2}$, \\ Ellen Chirwa $^{1}$ \\ ${ }^{1}$ University of Malawi, Kamuzu College of Nursing, Blantyre, Malawi; \\ "Corresponding Author: ukafula@yahoo.com, ursulakafulafula@kcn.unima.mw \\ ${ }^{2}$ New York University, New York, USA \\ ${ }^{3}$ Boston College of Nursing, Boston, USA
}

Received 26 June 2013; revised 30 July 2013; accepted 15 August 2013

Copyright (C) 2013 Ursula K. Kafulafula et al. This is an open access article distributed under the Creative Commons Attribution License, which permits unrestricted use, distribution, and reproduction in any medium, provided the original work is properly cited.

\section{ABSTRACT}

Rates of exclusive breastfeeding in Malawi remain low despite the acknowledged benefits of exclusive breastfeeding for the infant's wellbeing and the prevention of mother-to-child transmission of HIV. Creating an environment supportive of exclusive breastfeeding is critical to increase the rate of exclusive breastfeeding among HIV-positive mothers. However, little is known on factors that influence the environment within which HIV-positive mothers in Malawi practise exclusive breastfeeding. Therefore, the exploratory qualitative study on which this article is based was conducted at the Chatinkha maternity unit of Queen Elizabeth Central Hospital in Malawi from April 16, 2009 to May 8, 2009 to explore perceived practice environment related barriers to exclusive breastfeeding among HIVpositive mothers. Data were obtained through indepth interviews with 16 purposively selected breastfeeding HIV-positive mothers between 18 and 35 years old and two focus group discussions with women of unknown HIV status. Semistructured interview and focus group guides were utilised. Content analysis of data was done. Five main themes emerged regarding factors that may influence the environment within which exclusive breastfeeding was practised: 1) availability of resources; 2) societal norms and cultural practices; 3) mother-baby proximity; 4) health workers' attitudes and 5) disclosure of the mothers' HIV status. A multi-sectoral approach to promote exclusive breastfeeding is suggested. This should include community involvement because it is in the community where breastfeeding norms and cultural practices associated with breastfeeding are propagated.

Keywords: Barriers; Practice Environment; Exclusive Breastfeeding; HIV-Positive Mothers; Malawi

\section{INTRODUCTION}

In Malawi, it is estimated that the median duration of exclusive breastfeeding is 3.7 months [1] which falls short of the recommendations by the World Health Organization as well as the Malawi Ministry of Health that mothers exclusively breastfeed for the first six months of the child's life. This sub-optimal practice in exclusive breastfeeding is not unique to Malawi. Although $89.4 \%$ to $99 \%$ of mothers in the Sub-Saharan African countries breastfeed their children, with Uganda and Malawi having the highest rate of $99 \%$ [2-5], the practice of exclusive breastfeeding (defined as feeding an infant only breast milk from its mother) remains low. In some parts of Malawi, rates are as low as $19 \%$ at one month, and less than $5 \%$ at six months [6-10]. This is true regardless of whether the mother is HIV-positive $[8,9]$ or HIV-negative $[6,7,10]$.

Breastfeeding is a significant mode of mother-to-child transmission (MTCT) of HIV [11]. MTCT of HIV accounts for approximately $25 \%$ of new infections in Malawi [12]. The risk of MTCT of HIV through breastfeeding increases if mixed feeding (feeding an infant other liquids or solids in addition to breast milk) is practiced. Mixed feeding during the first six months of an infant's 
life carries a four to tenfold increased risk of MTCT of HIV compared to exclusive breastfeeding [13,14]. This is the case because mixed feeding increases the risk of diarrhoea to an infant due to the danger associated with the use of unsafe water during the constitution of powdered infant formula and possible contamination of non-breast milk feeds. Diarrhoea alters the infant's gastro-intestinal integrity and may, therefore, facilitate MTCT of HIV in mixed-fed infants [15]. Benefits of exclusive breastfeeding for the first six months for mothers who are poor outweigh those of replacement feeding (feeding an infant with artificial milk only) despite replacement feeding being the ideal way of feeding infants of HIV-positive mothers [16]. It is, therefore, important that exclusive breastfeeding be promoted among HIV-positive mothers living in resource-poor settings.

Previous studies conducted in Malawi have highlighted a variety of factors that may deter mothers from exclusively breastfeeding their infants. These factors include: poor socioeconomics [10], delivering in rural health facilities [17], beliefs about breast milk being insufficient and the high demands of EBF on the mother's body [7, 18], and the prevailing pre-lacteal feeding practices $[7,10$, 17]. Only Bentley et al. [18] included HIV-positive women in their study who believed that exclusive breastfeeding strains mothers' bodies and may enhance the progression of HIV infection to full-blown AIDS.

Breastfeeding is a behaviour that is rooted in societal norms and practices [19]. In Malawi, where exclusive breastfeeding is not the norm, mothers who choose to exclusively breastfeed may face challenges. Furthermore, HIV-positive mothers are in a more awkward situation than their counterparts who are HIV-negative because of the stigma associated with HIV infection and how these mothers might feed their infants. Creating a supportive environment within which an HIV-positive mother would practise exclusive breastfeeding may therefore be critical in increasing the rate of exclusive breastfeeding. Given the inadequate literature on factors that may hinder HIVpositive mothers from exclusively breastfeeding their children, this study was conducted to explore perceived practice environment related barriers to exclusive breastfeeding among HIV-positive mothers. This was achieved through in-depth interviews with HIV-positive mothers and focus group discussions with women who were assistants/guardians to maternity patients at the time of the study. This article presents the findings, discussion and conclusions drawn from the study and provides relevant recommendations.

\section{METHODS}

The study was exploratory and utilised qualitative methods to explore and gain in-depth knowledge and understanding of practice environment related barriers to ex- clusive breastfeeding among HIV-positive mothers in Blantyre, Malawi. Little is known about factors that may influence the environment within which exclusive breastfeeding takes place for the HIV-positive mothers in Blantyre, therefore an exploratory qualitative design was appropriate for this study.

\subsection{Study Setting}

This study was conducted at the Queen Elizabeth Central Hospital (QECH) maternity unit in Blantyre, Malawi. Participants were recruited from the postnatal wards, the postnatal clinic, and the Cotrim clinic (a clinic that provided early infant HIV screening and cotrimoxazole to HIV-exposed infants at the time of this study) within the QECH maternity unit. Blantyre is one of the districts in the Southern Region of Malawi with a population of 1.01 million, $68 \%$ of whom live within the urban area [20]. Blantyre is one of the districts in Malawi with high prenatal care coverage. Seventy per cent of women deliver in public health facilities [4]. It has organised services for PMTCT of HIV and prenatal HIV testing is an important gateway to these services. Availability of PMTCT services provided access for the study of HIV-positive postnatal mothers without the researchers testing the participants for HIV. QECH is a public hospital which functions both as a district hospital for Blantyre and as a referral hospital for the Southern Region of Malawi. It is also a teaching hospital for different health related professions.

\subsection{Sample}

A purposive sample of 27 women: 16 HIV-positive mothers and 11 women with unknown HIV status participated in this study. The sample size for the HIV-positive mothers was determined based on data saturation [21]. Since HIV-positive mothers who had children of less than six months of age were appropriate informants for the target population they were representing on issues concerning exclusive breastfeeding, data saturation was achieved with a relatively small sample [21] of 16 participants. Women with unknown HIV status were included in this study in order to represent possible views of HIV-negative mothers, and potential mothers and mothers-in-law of HIV-positive mothers on exclusive breastfeeding. The present study did not include relatives of the participating HIV-positive mothers, who would have been ideal informants for this study, because of the stigma associated with HIV infection and to maintain the privacy of the participants since some of them had not yet disclosed their HIV status to their relatives and significant others for fear of being stigmatised.

\subsection{Inclusion and Exclusion Criteria}

To participate in this study, the HIV-positive mothers 
had to be at least 18 years old; breastfeeding a single child that was less than six months old; able and willing to give informed consent; and able to understand and speak Chichewa (the vernacular). The HIV-positive breastfeeding mothers who were sick with AIDS, tuberculosis or other medical conditions were excluded from the study. To qualify for the "women with unknown HIV status" category, one had to be: at least 18 years old; of unknown HIV status; a personal assistant/guardian to a maternity patient at the time of the study; a mother with experience of breastfeeding a baby and not related to the participating HIV-positive mothers.

\subsection{Recruitment}

To access participants, the principal researcher first obtained permission from the Director of QECH and the head of the obstetrics and paediatric departments of QECH. Next, the researcher introduced the study to the Nursing Administrator of QECH and the matron of the Chatinkha maternity unit at $\mathrm{QECH}$ before commencing participant recruitment. One nurse-midwife, who was not working at QECH, was contracted as a research assistant (RA) to conduct participant recruitment and to assist with audiotape recording of focus group discussions (FGDs). The researcher made sure that the RA was oriented to and passed the basic United States of America National Institute of Health human subjects training prior to conducting participants' recruitment. The RA introduced the study to potential participants in the hospital's waiting areas. Recruitment scripts were utilised in order to maintain uniformity of information. Recruitment scripts were sensitively structured because they could influence the relationship between the potential participants and the researcher which begins the moment potential participants hear about the study. Although third parties may be used to gain access to potential participants, they should be utilised as little as possible to make actual contact with them [22]. This is why the researcher utilised third parties only to introduce the study and not for the actual contact with potential participants.

HIV-positive mothers were recruited from the PMTCT programme at QECH. Both paying and non-paying postnatal HIV-positive mothers were recruited. The recruitment procedure did not require any HIV testing and screening because all the mothers who were enrolled in the PMTCT programme had already been tested for HIV. Those who were interested and met the inclusion criteria met the researcher in a private quiet room where informed consent was obtained and in-depth interviews (IDIs) conducted. For the women with unknown HIV status, the RA recruited them among the female assistants (guardians) to maternity patients in the guardian lounge of Chatinkha maternity unit. Focus group discussions (FGDs) were scheduled for those who were interested and met the inclusion criteria.

\subsection{Data Collection}

Data were collected between 16 April 2009 and 8 May 2009. The researcher and the RA conducted the two FGDs while the researcher conducted the 16 IDIs in a room designated for the study. It was situated within the postnatal ward where recruitment was also done. This helped to keep data collection conditions constant. Oneon-one IDIs allowed HIV-positive mothers to participate in this study while maintaining privacy related to their HIV status from other participants. Two FGDs of women with unknown HIV status were conducted to complement the interviews. The two focus groups (FGs) were comprised of five and six participants respectively. Literature on FGD indicates that participant size of between four and 12 is adequate with the ideal size being between five and 10 participants [23-26]. Therefore, the size of the two FGs for this study was appropriate and acceptable.

A semi-structured IDI and FGD guide which included open-ended questions and prompts about facilitators and barriers to EBF was utilised. For this article, data came from two questions: 1) What makes it easier for HIV-positive mothers to exclusively breastfeed their babies? 2) What makes it harder for HIV-positive mothers to exclusively breastfeed their babies? These questions were rephrased in order to use them for interviewing HIV-positive mothers. Probes for areas like resources, commitment of HIV-positive mothers, knowledge and experience of exclusive breastfeeding, and socio-cultural issues such as cultural practices were included in the guides to help elicit more information from participants. Each audio-taped IDI and FGD took approximately one hour.

\subsection{Data Management and Analysis}

The audio-recorded information was transcribed verbatim and translated from Chichewa (the vernacular) to English. Observational notes were incorporated into the transcriptions. Data analysis was done manually and concurrently with data collection in order to identify and correct errors during the subsequent interviews and focus group discussion, and to improve the guides by incorporating issues that might have come up. Thematic content analysis was utilised [27]. At the beginning of the analysis, the researcher read through one transcript quickly to get a sense of what was in it, and then read it again, this time closely and critically to identify codes that captured meaning [28]. The researcher discussed the initial coding scheme with experts in qualitative data analysis for validation of the accuracy of the codes. After incorporating any comments from the experts, the final coding scheme was then used to code the remaining transcripts from the 
IDIs and the FGDs. The codes were organised into mutually exclusive categories based on their similarities [27]. The different categories were then brought together to develop overarching themes represented by the different categories [29] which were presented as findings. A detailed record of all what was done was kept to serve as audit trail.

\subsection{Ethical Considerations}

The New York University Committee on Activities Involving Human Subjects and the Malawi College of Medicine Research and Ethics Committee approved this study. In addition, permission to access participants was granted by the Director of QECH, the head of obstetrics and gynaecology, and the head of the paediatric departments at QECH. Written informed consent was obtained from each participant prior to participation. To avoid coercion, health care providers of HIV-positive mothers did not directly participate in this study. To help reduce stigmatisation of FGD members, names were not used when sharing information. Personal experiences were shared as if they were of someone else. Numbers were assigned to participants to be used when quoting information during data analysis.

\section{TRUSTWORTHINESS}

The scientific rigor of the data was maintained by addressing Lincoln and Guba's (1985) 4-item criteria for evaluating the trustworthiness of qualitative data, namely credibility, dependability, confirmability and transferability. Credibility was assured through prolonged immersion of the researcher into the situation, and triangulation both by methods and source of data. Additionally, participants were encouraged through probes to share their perspectives without aiming for consensus. Dependability was assured by: 1) using the same semistructured interview guide with each IDI and FGD although allowing for flexibility; 2) providing similar conditions with each group of participants; 3) preparing transcripts promptly; and 4) using direct quotes (the English translations of Chichewa quotes) when presenting findings. Confirmability was assured by providing a detailed description of what was done and decisions made, which later served as an audit trail. Finally, transferability was assured by describing the sample and setting in detail so that potential appliers can make transferability decisions [21,30].

\section{FINDINGS}

\subsection{Demographic Characteristics}

Sixteen HIV-positive mothers participated in this study. These mothers were breastfeeding infants whose ages ranged from two days to 24 weeks. The ages of the mothers ranged from 18 to 35 years with a mean age of 27.7 years (Standard deviation of 5.326). In addition, 11 women with unknown HIV status participated in this study. Two of them were in the age range of 30 to 35 years while nine were between 45 and 55 years. The two women in the age range of 30 to 35 years were still breastfeeding their children at the time of this study. One was breastfeeding a 20 weeks ( 5 months) old infant while the other woman was breastfeeding a 48 weeks (12 months) old child. Details of the demographic data of the participating HIV-positive mothers are summarized in Table 1.

\subsection{Practice Environment Related Barriers to Exclusive Breastfeeding}

Several themes of factors related to an exclusive breastfeeding environment emerged from the IDIs and FGDs. These themes were categorised as: availability of resources; societal norms and cultural practices; motherbaby proximity; health workers' attitudes and disclosure of one's HIV status.

\subsubsection{Availability of Resources}

Three sub-themes emerged from the availability of resources which were: adequacy of maternal diet; availability of money and exclusive breastfeeding support services and information.

1) Adequacy of maternal diet

Table 1. Characteristics of HIV-positive mothers for in-depth interviews.

\begin{tabular}{ccccccc}
\hline No & Age & Parity & $\begin{array}{c}\text { HIV } \\
\text { disclosure to } \\
\text { husband } \\
\text { or mother }\end{array}$ & $\begin{array}{c}\text { Mother } \\
\text { working? }\end{array}$ & $\begin{array}{c}\text { Mother } \\
\text { has }\end{array}$ & $\begin{array}{c}\text { Age of } \\
\text { baby being } \\
\text { breastfed }\end{array}$ \\
\hline 1 & 18 & 1 & No & No & No & 2 days \\
2 & 18 & 1 & No & No & No & 2 days \\
3 & 22 & 1 & Yes & No & No & 4 days \\
4 & 23 & 1 & No & No & No & 3 days \\
5 & 26 & 1 & Yes & No & No & 2 days \\
6 & 32 & 1 & Yes & Yes & Yes & 6 wks \\
7 & 33 & 2 & Yes & No & No & 7 wks \\
8 & 26 & 2 & Yes & No & No & 24 wks \\
9 & 30 & 3 & No & No & No & 2 days \\
10 & 34 & 2 & Yes & No & No & 2 days \\
11 & 27 & 2 & No & No & No & 4 days \\
12 & 35 & 6 & No & No & Yes & 4 days \\
13 & 29 & 1 & Yes & Yes & Yes & 6 wks \\
14 & 27 & 2 & No & Yes & Yes & 2 wks \\
15 & 32 & 3 & Yes & No & No & 6 wks \\
16 & 31 & 1 & No & Yes & Yes & 6 wks \\
\hline
\end{tabular}

Key: MSCE = Malawi School Certificate of Education (academic qualification offered at the end of four years of successful secondary school education); wks = weeks. 
The sub-theme of adequacy of maternal diet emerged from both groups of participants who viewed maternal diet as an important factor in successful exclusive breastfeeding. Adequacy of maternal diet featured commonly in the narrations from the participating HIV-positive mothers. Nearly all of these participants reported that they would need adequate diet in order to successfully breastfeed their babies.

It is the availability of appropriate food for me that can help me to exclusively breastfeed my baby (Interviewee $_{12}$ ).

Some people are from the village. They might want to exclusively breastfeed their babies but they can only manage to have little food in the morning and evening. For such mothers, it is difficult to exclusively breastfeed because of lack of appropriate and adequate food in their homes (Interviewee ${ }_{6}$ ).

According to these participants, mothers need an adequate diet to produce sufficient milk for their babies.

The mother should eat adequate food all the time so that her body can produce adequate milk to satisfy the baby (Interviewee ${ }_{2}$ ).

Similarly, participants from the FGDs reported that an HIV-positive mother needs an adequate amount of food to successfully breastfeed her baby exclusively.

This mother who is HIV-positive needs to eat food of all six groups during this time so that she can breastfeed her baby well $\left(\mathrm{FG}_{2} \mathrm{P}_{3}\right)$.

2) Availability of money

The sub-theme of availability of money emerged only from the interviews with HIV-positive mothers. These mothers, most of who were not working, reported that availability of money would help HIV-positive mothers to exclusively breastfeed their babies because they would be able to purchase food.

Also money for buying food can help her to exclusively breastfeed (Interviewee ${ }_{12}$ ).

Furthermore, another HIV-positive mother reported that money could be used to pay for transport to bring the baby to its mother at the mother's work-place. This would help a working mother to breastfeed her baby even when she is at her work-place.

If one has to go to work and has money for transport, relatives can bring the baby to her to breastfeed (Interviewee 13 ).

3) Exclusive breastfeeding support services and information

The findings revealed that availability of appropriate breastfeeding advice from health workers would help HIV- positive mothers to exclusively breastfeed. This narration came from both IDIs and FGDs. However, one of the participants also reported that the advice would be useless if the one being advised does not follow it.

By giving me advice, for example, health workers say that eating cassava and soaked rice every morning helps the mother to have adequate milk in her breasts. I think I will try it. (Interviewee ${ }_{2}$ ).

Giving me advice about breastfeeding would help me to exclusively breastfeed. But I also need to follow the advice that I have been given at the hospital for it to help me (Interviewee 4 ).

It is important that the breastfeeding advice given to these mothers be consistent for them to benefit from it. This was evident through the response of one HIV-positive mother who repeatedly pointed out the problem that inconsistent breastfeeding information could cause. She reported having received conflicting information on breastfeeding from health workers and fellow mothers and pointed out that it could be confusing to the HIVpositive mothers as it is difficult to know what is correct.

I have heard but not from health workers but from mothers. They say that HIV-positive mothers should breast-feed their babies only for three months beyond which the baby can contract the HIV virus from its mother. But this is not what health workers recommend. Health workers say we should breastfeed for six months (Interviewee ${ }_{16}$ ).

Later, this participant warned against listening to those who are not health workers as this could make exclusive breastfeeding harder.

Listening to what people who are not health workers say can prevent me from exclusively breastfeeding my baby. Health workers say that we should breastfeed for six months while other people say three months which is confusing. One can do things that are not good for self and the baby. We follow the advice that we are given by health workers (Interviewee ${ }_{16}$ ).

Participants from the FGDs concurred with the participating HIV-positive mothers on the importance of breastfeeding support from family members and health workers in promoting exclusive breastfeeding.

There are some husbands who remind their wives about breastfeeding. For example, they might ask their wives whether they have breastfed their babies after coming back from fetching water from a well. Some mothers too do remind their daughters about breastfeeding $\left(\mathrm{FG}_{1} \mathrm{P}_{1}\right)$.

The doctor too can encourage her to breastfeed her baby $\left(\mathrm{FG}_{1} \mathrm{P}_{3}\right)$. 


\subsubsection{Mother-Baby Proximity}

Findings from both groups of participants revealed that it is important for mothers to be close to their babies in order to successfully breastfeed exclusively. Two subthemes emerged from the mother-baby proximity: mother returning to work and rooming-in.

1) Mother returning to work

According to the participants, maternal employment reduces the amount of time mothers spend with their babies, especially when mothers return to their work unless special mechanisms are put in place to help keep the two together. In her narration one HIV-positive mother suggested a strategy of bringing the baby to its mother's work-place to breastfeed.

If one has to go to work and has money for transport, relatives can bring the baby to her to breastfeed (Interviewee ${ }_{13}$ ).

Some participants from the FGDs were pessimistic about the success of working mothers on exclusive breastfeeding because maternal employment does not allow mothers adequate time to be with their babies. However, as a solution to this problem, one participant suggested that breastfeeding mothers should be given maternity leave so that they could breastfeed their babies longer before resuming their work.

Staying closer to her baby, not leaving the baby alone can help. For example, a working mother should be given a holiday to stay at home so that she can breastfeed her baby $\left(\mathrm{FG}_{1} \mathrm{P}_{1}\right)$.

In support of the idea, other participants had this to say:

On that point, it is important that the mother should stay with her baby. Since some mothers employ baby sitters but for a mother to be able to exclusively breastfeed her baby, she needs to stay close to her baby $\left(\mathrm{FG}_{1} \mathrm{P}_{4}\right)$.

If the mother is working, she cannot manage to exclusively breastfeed her baby because she is at work when the baby needs to breastfeed $\left(\mathrm{FG}^{\mathrm{P}} \mathrm{P}_{3}\right)$.

So if she loves her job more than her baby she will definitely fail to exclusively breastfeed [said this in high tone of voice] $\left(\mathrm{FG}_{1} \mathrm{P}_{6}\right)$.

\section{2) Rooming-in}

Exclusive breastfeeding demands that the mother and her baby be together most of their time. To help keep the two together, some tribes in Malawi have cultural practices that encourage breastfeeding mothers to sleep together with their babies. Although these practices are normally intended to promote breastfeeding by encouraging mothers to be together with their babies, one HIV-positive mother reported that the practice may also nega- tively influence exclusive breastfeeding because expecting breastfeeding mothers to sleep together with their babies at all times may not be possible or practical.

I have heard of one belief which says that if a breastfeeding mother sleeps away from her baby for whatever reason, she should not breastfeed the baby when she comes back until she has chewed some traditional medicine for fear of Tsempho [Tsempho is the name of the disease that the baby would suffer if postnatal sexual abstinence is violated]. I don't know how practical this is (Interviewee ${ }_{11}$ ).

\subsubsection{Health Workers' Attitudes}

Health workers have the authority in the dissemination of health related information to clients that may come under their care. For these clients to take heed of the information given, a cordial relationship needs to exist between the two. However, poor attitudes on the part of the health workers may distance clients from them, thereby preventing them from benefiting from the intended advice. This came out clearly from the participants' narratives. Participants reported that it would be easier for HIV-positive mothers to exclusively breastfeed if health workers did not show negative attitudes towards them.

Also by having health workers not showing negative attitudes to mothers who choose to breastfeed their babies (FG1P2).

\subsubsection{Societal Norms and Cultural Practices}

Two sub-themes emerged from the societal norms and cultural practices: giving a baby expressed breast milk from a cup and postnatal sex and sexual abstinence.

1) Giving a baby expressed breast milk from a cup

The sub-theme of giving a baby expressed breast milk from a cup emerged from HIV-positive mothers only. Participants who expressed their breast milk and fed their baby using a cup helped the babies to feed on their mothers' milk without the mothers being physically present, since anyone could feed the baby. This could help working mothers to continue exclusively breastfeeding their babies while being away from their babies. One participant had this to say:

Expressing breast milk into a cup or a bottle and feeding the baby to make sure that the baby is exclusively breastfed when the mother is away would make exclusive breastfeeding easier because babies could be fed with their mothers' milk even in absence of their mothers (Interviewee ${ }_{15}$ ).

However, this same participant later expressed her concern about the safety of the expressed breast milk for the baby. According to her, expressed breast milk could easily become contaminated and put baby at risk of in- 
fections.

Although I have said that expressing breast milk can help, breast milk that is expressed in a cup or a bottle can easily get contaminated with germs and make the baby sick (Interview 15 ).

2) Postnatal sex and sexual abstinence

Participants reported that some cultures in Malawi expect breastfeeding mothers to abstain from sex for a prescribed period of time ranging from six to nine months. During this time, some mothers are completely separated from their husbands. Some participants felt that such a practice could help mothers to exclusively breastfeed their babies.

When the mother is discharged from the hospital, she and the baby go into postnatal seclusion ("Chikuta" in the native language). At times this means going away from the husband in case they cheat on the practice. We wait before we can sleep with the husband. Some stay in the postnatal seclusion for six months, others seven months but we stay for eight months (Interviewee ${ }_{11}$ ).

For those cultures that do not practise postnatal seclusion, mothers could share the same house with their husbands but are expected to abstain from sex until the baby has reached a certain age as stipulated by their tribal practices.

According to my cultural belief, the mother should breastfeed the baby until when the baby is two years old. Another belief is that the mother should not sleep [not have sex] with the husband until the baby is nine months old although you are in the same house (Interviewee ${ }_{8}$ ).

To make sure that mothers abide by postnatal sexual abstinence, tribal members following such practices are made to believe that the baby could become sick if its parents violate the practice of sexual abstinence. In fear of making their baby sick, the parents are forced to abide to the practice. When probed on what would happen if the mother had sex with her husband before the end of the eight months, one mother said:

The bay gets Tsempho. The baby is unhappy with a weak body and swells up (Interviewee ${ }_{11}$ ).

However, other participants viewed postnatal sexual abstinence as a barrier to exclusive breastfeeding especially for those who might not manage to abstain, as illustrated by the following quotes:

Some say don't sleep with the husband while breastfeeding because the baby will feed on hot milk that can make the baby sick. So the mother can just stop breastfeeding the baby if she can't manage to con- tinue abstaining (Interviewee $)_{1}$.

A belief that can make it harder for mothers to exclusively breastfeed is that if a breastfeeding mother and her husband have sex, the baby will develop a childhood disease called "Tsempho". But I don't believe in it (while giggling) (Interviewee ). $^{\text {. }}$

\subsubsection{Disclosure of One's HIV Status}

This theme emerged from the FGDs only. Participants reported that failure on the part of mothers to disclose their HIV status may make it difficult for them to exclusively breastfeed. It is difficult for a mother to tell her significant others why she needs to exclusively breastfeed her baby if she has not told them that she is HIVpositive. Consequently, such a mother may not receive appropriate support towards exclusive breastfeeding.

Because one cannot disclose one's HIV status it will then be difficult to say why the baby should be exclusively breastfed if you haven't told your husband you have $\mathrm{HIV}\left(\mathrm{FG}_{2} \mathrm{P}_{3}\right)$.

Because people hide their sero status, it is difficult to offer help and encouragement if one has not told those you live with $\left(\mathrm{FG}_{2} \mathrm{P}_{1}\right)$.

\section{DISCUSSION}

This study has revealed several factors that may positively and negatively influence the environment within which HIV-positive mothers practice exclusive breastfeeding. Five main themes emerged from these factors: availability of resources, societal norms and cultural practices, mother-baby proximity, health workers' attitudes and disclosure of one's HIV status. Participants, especially the HIV-positive mothers, repeatedly expressed the need for them to eat adequate and appropriate food in order to produce sufficient milk for successful exclusive breastfeeding. Consistent with this finding was that in Kenya [31], Swaziland, South Africa and Namibia [32] HIV-positive women also believed that an adequate diet was needed for women to produce sufficient milk. Although no evidence exists to support a direct relationship between maternal dietary intake and amount of breast milk production, the finding from the current study may be of great significance in promoting exclusive breastfeeding considering the fact that most of the HIV-positive mothers that choose to breastfeed their babies in Malawi and other developing countries are poor [33-34] and may therefore have problems in finding adequate and appropriate food for themselves and their families. These women are likely to be distressed because each day they will be worried about what to feed their families and themselves. In an attempt to find money for buying food, these women may be involved in piece work outside their homes. This may reduce the amount of quality time 
they spend with their babies and consequently reduce the duration of exclusive breastfeeding. One of the participants strongly put it: "If the mother is working, she cannot manage to exclusively breastfeed her baby because she is at work when the baby needs to breastfeed." This finding is consistent with those from previous studies [35-37] that revealed a significant association between maternal employment and reduced exclusive breastfeeding duration. Furthermore, the distress associated with constant worry about food insecurity may affect milk letdown, breast emptying and subsequent milk production [38,39]. Improving HIV-positive mothers' availability of food may therefore reduce distress associated with food insecurity and the chances of the mothers being away from their babies in search of food. In turn, this may positively influence rates of EBF.

Participants in the current study also reported that appropriate breastfeeding advice and support from health workers and family members would help HIV-positive mothers to exclusively breastfeed their babies. Strong evidence exists on the positive impact of breastfeeding support on the duration of EBF. Regardless of who provided the breastfeeding support, interventions that provided both breastfeeding knowledge and ongoing one-toone breastfeeding support and counselling significantly increased the duration of exclusive breastfeeding [40-44]. In a study that was conducted in the Northern Region of Malawi [7] it was revealed that paternal grandmothers play an important role in issues concerning infant feeding practices. To have a positive effect on rates of EBF, breastfeeding support interventions in such cultures need to include grandmothers as breastfeeding support providers.

Besides family members, health workers are another important source of health related (including breastfeeding) information and support for mothers. This was true with regard to participants in the current study who reported that health workers such as doctors and nurses could also provide appropriate breastfeeding information and support to HIV-positive mothers. However, these participants also commented that it would be easier for HIVpositive mothers to exclusively breastfeed if these health workers did not show negative attitudes towards them. This finding is important for practical reasons in creating an environment that is conducive to open communication because any negative attitudes health workers may display towards HIV-positive mothers could deter them from further seeking information from them [45]. This may have a negative impact on rates of EBF among these mothers.

Societal norms and practices play an important role in breastfeeding [19]. Although not supported by data from the FGDs, participating HIV-positive mothers reported cultural beliefs and practices surrounding postnatal sex and sexual abstinence as possible barriers to EBF. Despite the intended merits of these cultural practices in promoting culturally accepted child rearing, participants voiced their concerns about the unrealistic expectations imposed on breastfeeding mothers such as to sleep together with their babies at all times. It was clear from the current study that this expectation could not be met by working mothers whose work requires long distance travelling and night shifts. This may partly explain why participants in one FG felt strongly that working mothers cannot manage to exclusively breastfeed their babies because the mother may not be home when the baby needs to be breastfed. Consistent with these findings were the findings from previous studies from Sub-Saharan Africa [19, $35,46]$ that also reported cultural beliefs and practices that could make it difficult for mothers to exclusively breastfeed their babies. In Malawi, studies have revealed that prelacteal feeding $[7,10,17]$ and great powers invested in paternal grandmothers on issues concerning infant feeding are some of the cultural practices and norms that may influence EBF. Cultural norms and practices such as these need to be addressed in EBF promotion activities because they could deter mothers from exclusively breastfeeding their babies despite their intended merits in promoting breastfeeding duration. This is particularly true in Malawi and other similar cultures with deeply engrained cultural traditions.

\section{CONCLUSION}

The aim of the study on which this article is based was to explore barriers to exclusive breastfeeding among HIVpositive mothers in Blantyre, Malawi. In-depth interviews with HIV-positive mothers who were breastfeeding their infants were conducted using a semistructured interview guide. To complement the data, two focus group discussions were also conducted with women whose HIV statuses were unknown. The findings have revealed multiple aspects of barriers to exclusive breastfeeding which may influence rates of exclusive breastfeeding among HIV-positive mothers. Factors that may influence the environment within which HIV-positive mothers exclusively breastfeed their infants have been highlighted. Commonly reported factors that may render the environment unfavourable to exclusive breast-feeding for HIV-positive mothers were availability of resources and cultural expectations surrounding infant feeding. Participants strongly felt that HIV-positive mothers require an adequate diet in order to produce sufficient breast milk.

Although the findings of the current study may be applicable to other HIV-positive mothers in Blantyre, Malawi, their applicability to HIV-positive mothers of different socio-economic status may be limited because participants were drawn from one site that caters mainly for low-income women. It is not known what HIV-positive 
mothers of high social economic statuses would perceive as barriers to exclusive breastfeeding. Further research involving a diverse population of HIV-positive mothers and their significant others may be necessary to explore the phenomenon of practice environment related barriers to exclusive breastfeeding in Malawi.

Despite the limitations to this study which have been described, the findings suggest the need to involve significant others in issues surrounding infant feeding and rearing. Ongoing individual and couple counselling to promote the adoption of exclusive breastfeeding should be encouraged at the one and six weeks' postnatal checkup where mothers could get appropriate breastfeeding support and information. Referral systems for dietary supplementation for mothers who may not have access to adequate nutrition should be put in place at each hospital that runs a prevention of mother-to-child transmission of HIV programme. In conclusion, there is a need for community involvement because it is in the community where breastfeeding norms and cultural practices associated with breastfeeding are propagated.

\section{ACKNOWLEDGEMENTS}

We wish to acknowledge New York University School of Nursing and Kamuzu College of Nursing for the financial support towards the senior author's postgraduate studies. The study on which this article is based was part of the senior author's Doctor of Philosophy degree in Nursing Research and Theory Development at New York University. We would also like to acknowledge all mothers and assistants to maternity patients at Queen Elizabeth Central Hospital who participated in this study.

\section{REFERENCES}

[1] National Statistical Office (NSO) [Malawi] and ORC Macro (2011) Malawi demographic and health survey2010. Nso and ORC Macro, Calverton.

[2] Nankunda, J., Tumwine, J.K., Soltvedt, A. Semiyaga, N., Ndeezi, G. and Tylleskär, T. (2006) Community based peer counsellors for support of exclusive breastfeeding: Experiences from rural Uganda.

http://www.internationalbreastfeedingjournal.com/content $\underline{/ 1 / 1 / 19}$

[3] Orne-Gliemann, J., Mukotekwa, T., Miller, A., Perez, F., Glenshaw, M., Nesara, P., et al. (2006) Community-based assessment of infant feeding practices within a programme for prevention of mother-to-child HIV transmission in rural Zimbabwe. Public Health Nutrition, 9, 563-569.

[4] National Statistical Office (NSO) [Malawi], and ORC Macro (2005) Malawi demographic and health survey-2004. Nso and ORC Macro, Calverton.

[5] World Health Organisation (WHO) (2006) Antiretroviral drugs for treating pregnant women and preventing HIV infection in infants: Towards universal access. Recommendations for a public health approach. Geneva.

[6] Kalanda, B.F., Verhoeff, F.H. and Brabin, B.J. (2006)
Breastfeeding and complementary feeding practices in relation to morbidity and growth in Malawian infants. European Journal of Clinical Nutrition, 60, 401-407. doi:10.1038/sj.ejen. 1602330

[7] Kerr, R.B., Berti, P.R. and Chirwa, M. (2007) Breastfeeding and mixed feeding practices in Malawi: Timing, reasons, decision makers, and child health consequences. Food and Nutrition Bulletin, 28, 90-99.

[8] Omari, A.A.A., Luo, C., Kankasa, C., Bhat, G.J. and Bunn, J. (2003) Infant-feeding practices of mothers of known HIV status in Lusaka, Zambia. Health Policy and Planning, 18, 156-162. doi:10.1093/heapol/czg020

[9] Poggensee, G., Schulze, K., Moneta, I., Mbezi P., Baryomunsi, C., Harms, G., et al. (2004) Infant feeding practices in western Tanzania and Uganda: implications for infant feeding recommendations for HIV-infected mothers. Tropical Medicine and International Health, 9, 477 485.

[10] Vaahtera, M., Kulmala, T., Hietamen, A., Ndekha, M., Cullinan, T., Salin, M.L., et al. (2001) Breastfeeding and complementary feeding practices in Malawi. Acta Pcediatrica, 90, 328-332.

doi:10.1111/j.1651-2227.2001.tb00313.x

[11] De Cock, K.M., Fowler, M.G., Mercier, E., de Vincenzi, I., Saba, J., Hoff, E., et al. (2000) Prevention of mother-tochild HIV transmission in resource-poor countries. Journal of American Medical Association, 283, 1175-1182.

[12] National AIDS Commission (NAC) of Malawi (2009) Malawi HIV and AIDS extended National action framework 2010-2011 DRAFT. Malawi Government, Lilongwe.

[13] Coovadia, H.M., Rollins, N.C., Bland, R.M., Little, K., Coutsoudis, A., Bannish, M.L., et al. (2007) Mother-tochild transmission of HIV-1 infection during exclusive breastfeeding in the first 6 months of life: An intervention cohort study. Lancet, 369, 1107-1116. doi:10.1016/S0140-6736(07)60283-9

[14] Iliff, P.J., Piwoz, E.G., Tavengwa, N.V., Zunguza, C.D., Marinda, E.T., Nathoo, K.J., et al. (2005) Early exclusive breastfeeding reduces the risk of postnatal HIV-1 transmission and increases HIV-free survival. AID, 19, 699708. doi:10.1097/01.aids.0000166093.16446.c9

[15] Miller, M., Iliff, P., Stoltzfus, R.J. and Humphrey, J. (2002) Breastmilk erythropoietin and mother-to-child HIV transmission through breastmilk. The Lancet, 360, 1246-1248. doi:10.1016/S0140-6736(02)11277-3

[16] Coutsoudis, A., Coovadia, H.M. and Wilfert, C.M. (2008) HIV, infant feeding and more perils for poor people: New WHO guidelines encourage review of formula milk policies. Bulletin of the World Health Organization, 86, 210214.

[17] Kamudoni, P., Maleta, K., Shi, Z. and Holmboe-Ottesen, G. (2007) Infant feeding practices in the first 6 months and associated factors in a rural and semiurban community in Mangochi District, Malawi. Journal of Human Lactation, 23, 325-332.

[18] Bentley, M.E., Corneli, A.L., Piwoz, E., Moses, A., Nkhoma, J., Tohill, B.C., et al. (2005) Perceptions of the role of maternal nutrition in HIV-positive breastfeeding wo- 
men in Malawi. Journal of Nutrition, 135, 945-949.

[19] Yovsi, R.D. and Keller, H. (2003) Breastfeeding: An adaptive process. Ethos, 31, 147-171.

[20] Daza, P., Banda, R., Misoya, K., Katsulukuta, A., Gessner, B.D., Katsande, R., et al. (2006) The impact of routine infant immunisation with haemophilus influenzae type $b$ conjugate vaccine in Malawi, a country with high human immunodeficiency virus prevalence. Vaccine, 24, 62326239. doi:10.1016/j.vaccine.2006.05.076

[21] Polit, D.F. and Beck, C.T. (2008) Nursing research. Generating and assessing evidence for nursing practice. Wolters Kluwer/Lippincott Williams \& Wilkins, Philadelphia.

[22] Seidman, I.E. (1991) Interviewing as qualitative research. Teachers College, New York.

[23] Beyea, S.C. and Nicoll, L.H. (2000) Learn more using focus groups. AORN Journal, 71, 897-900. doi:10.1016/S0001-2092(06)62276-X

[24] Gulanick, M. and Keough, V. (1997) Focus groups: An exciting approach to clinical nursing research. Progress in Cardiovascular Nursing, 12, 24-29.

[25] Morgan, D.L. (1998) The focus group guidebook. Focus group kit1. Sage Publication, Thousand Oaks.

[26] Sim, J. (1998) Collecting and analyzing qualitative data: Issues raised by the focus group. Journal of Advanced Nursing, 28, 345-352. doi:10.1046/j.1365-2648.1998.00692.x

[27] Graneheim, U.H. and Lundman, B. (2004) Qualitative content analysis in nursing research: Concepts, procedures and measures to achieve trustworthiness. Nurse Education Today, 24, 105-112. doi:10.1016/j.nedt.2003.10.001

[28] Hsieh, H.F. and Shannon, S.E. (2005) Three approaches to qualitative content analysis. Qualitative Health Research, 15, 1277-1288.

[29] DeSantis, L. and Ugarriza, D.N. (2000) The concept of theme as used in qualitative nursing research. Western Journal of Nursing Research, 22, 351-372.

[30] Morrison-Beedy, D., Cote-Arsenault, D. and Feinstein, N.F. (2001) Maximizing results with focus groups: Moderator and analysis issues. Applied Nursing Research, 14, 48-53.

[31] Webb-Girard, A., Cherobon, A., Mbugua, S., KamauMbuthia, E., Amin, A. and Sellen, D.W. (2012) Food insecurity is associated with attitudes towards exclusive breastfeeding among women in urban Kenya. Maternal and Child Nutrition, 8, 199-214. doi:10.1111/j.1740-8709.2010.00272.x

[32] Buskens, I., Jaffe, A. and Mkhatswa, H. (2007) Infant feeding practices: Realities and mind sets of mothers in southern Africa. AIDS Care, 19, 1101-1109. doi:10.1080/09540120701336400

[33] International Baby Food Network, Africa (IBFAN Africa) (2007) HIV and infant feeding. https://www.ibfan-africa.org/blog4/hiv-and-infant-feeding/

[34] National AIDS Commission (NAC) of Malawi (2005) HIV/AIDS research strategy for Malawi 2005-2007. Design Printers, Lilongwe.
[35] Otoo, G.E., Lartey, A.A. and Perez-Escamilla, R. (2009) Perceived incentives and barriers to exclusive breastfeeding among periurban Ghanaian women. Journal of $\mathrm{Hu}$ man Lactation, 25, 34-41.

[36] Mamabolo, R.I., Alberts, M., Mbenyane, G.X., Steyn, N.P., Nthangeni, N.G., Delemane-Van De Waal, H.A., et al. (2004) Feeding practices and growth of infants from birth to 12 months in the central region of Limpopo Providence of South Africa. Nutrition, 20, 327-333. doi:10.1016/j.nut.2003.11.011

[37] Moffat, T. (2002) Breastfeeding, wage labor, and insufficient milk in peri-urban Kathmandu, Nepal. Medical Anthropology, 21, 207-230. doi:10.1080/01459740212902

[38] Jelliffe, D.B. and Jelliffe, E.F. (1978) The volume and composition of human milk in poorly nourished communities. A review. The American Journal of Clinical Nutrition, 31, 492-515.

[39] Dewey, K.G. (2001) Maternal and fetal stress are associated with impaired lactogenesis in humans. Journal of Nutrition, 131, 3012S-3015S.

[40] Aidam, B.A., Perez-Escamilla, R. and Lartey, A. (2005) Lactation counseling increases exclusive breast-feeding rates in Ghana. Journal of Nutrition, 135, 1691-1695.

[41] Mattar, C.N., Chong, Y.S., Chan, Y.S., Chew, A., Tan, P., Chan, Y.H., et al. (2007) Simple antenatal preparation to improve breastfeeding practice. Obstetric \& Gynecology, 109, 73-80.

[42] Noel-Weiss, J., Rupp, A., Cragg, B., Bassett, V. and Woodend, A.K. (2006) Randomization controlled trial to determine effects of prenatal breastfeeding workshop on maternal breastfeeding self-efficacy and breastfeeding duration. Journal of Obstetric, Gynecologic, and Neonatal Nursing, 35, 616-624.

[43] Su, L.L., Chong, Y.S., Chan, Y.H., Chan, Y.S., Fok, D., Tun, K.T., et al. (2007) Antenatal education and postnatal support strategies for improving rates of exclusive breastfeeding: Randomized controlled trial. British $\mathrm{Me}$ dical Journal, 335, 596. doi:10.1136/bmj.39279.656343.55

[44] Wolfberg, A.J., Michels, K.B., Shields, W., O’Campo, P., Bronner, Y. and Bienstock, J. (2004) Dads as breastfeeding advocates: Results from a randomized controlled trial of an educational intervention. American Journal of $\mathrm{Ob}$ stetrics and Gynecology, 191, 708-712. doi:10.1016/j.ajog.2004.05.019

[45] Painter, T.M., Dlaby, K.L., Matia, D.M., Lin, L.S., Sibailly, T.S., Kouassi, M.K., et al. (2004) Women's reasons for not participating in follow up visits before starting short course antiretroviral prophylaxis for prevention of mother to child transmission of HIV: Qualitative interview study. British Medical Journal, 329, 543-547. doi:10.1136/bmj.329.7465.543

[46] Kakute, P.N., Ngum, J., Mitchell, P., Kroll, K.A., Forgwei, G.W., Ngwang, L.K., et al. (2005) Cultural barriers to exclusive breastfeeding by mothers in a rural area of Cameroon, Africa. Journal of Midwifery \& Women's Health, 50, 324-328 